rev.relac.int.estrateg.segur.12(1):159-183,2017

\title{
ARGENTINA EN EL ESPACIO: POLÍTICA INTERNACIONAL EN RELACIÓN A LA POLÍTICA TECNOLÓGICA Y EL DESARROLLO INDUSTRIAL*
}

\author{
Daniel Blinder**
}

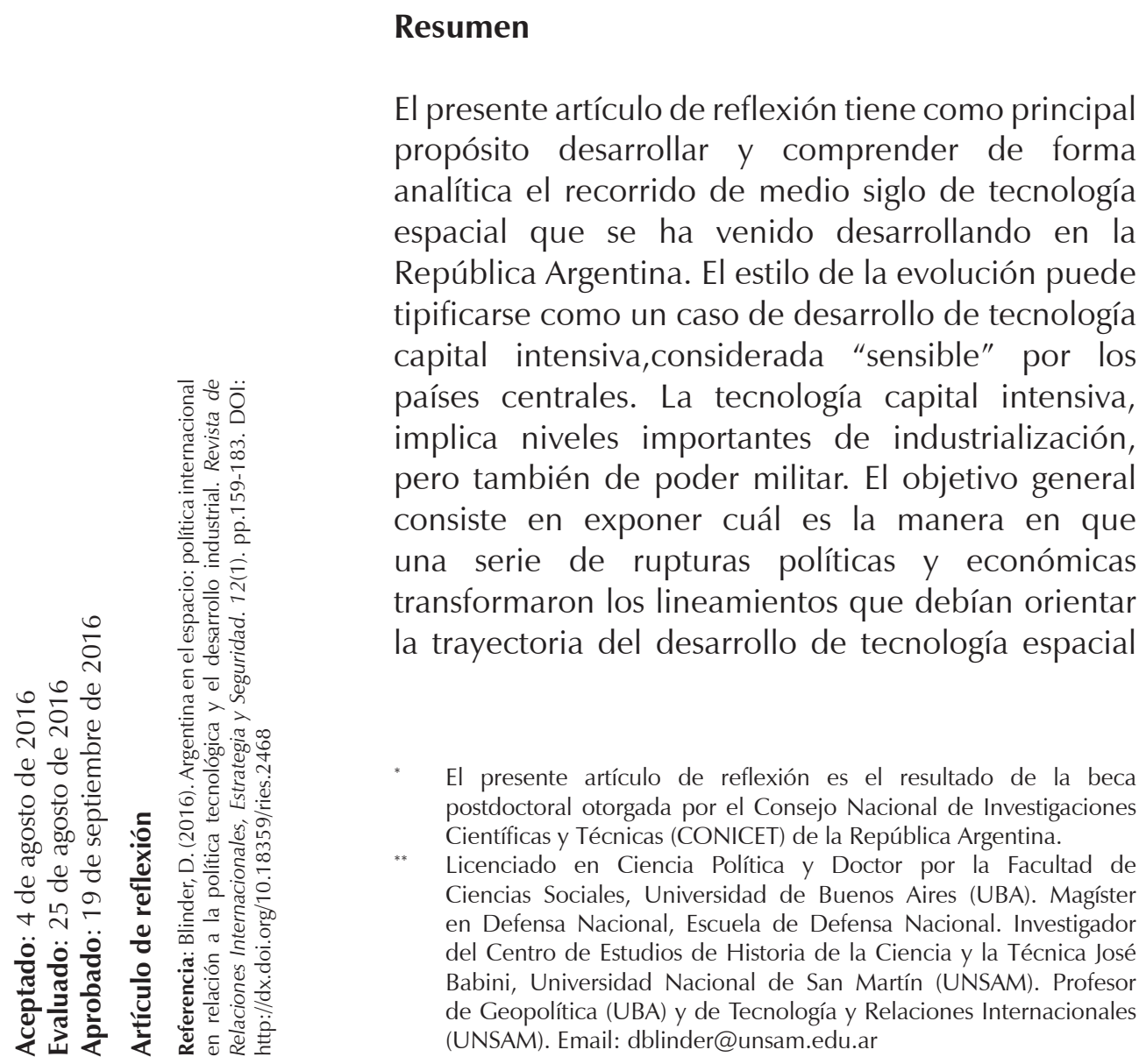


así como su aplicación en el uso civil y en el bélico. Igualmente, el artículo explica cómo funciona la disciplina de las Relaciones Internacionales a partir de una política tecnológica, permitiéndose mostrar los principales resultados de este sector transversal y supranacional.

Palabras Clave: Argentina, Tecnologías sensibles, Uso Dual, Tecnología Espacial

\title{
ARGENTINA IN SPACE: INTERNATIONAL POLITICS REGARDING TECHNOLOGICAL POLICIES AND INDUSTRIAL DEVELOPMENT
}

\begin{abstract}
This reflection article has as its main purpose to present and analytically understand the 50-years-long history of spatial technology been developed in the Republic of Argentina. The evolution style could be identified as a case of intensive capital technology development, considered by the central countries. Intensive capital technology implies important levels of industrialization, but also military power. The general objective is to elaborate on the way a series of political and economic breakdowns transformed the guidelines orienting the spatial technology developmental path, as well as its application for civil and military uses. Likewise, the article explains how the discipline of International Relations operates from a technological policy, showing the main outcomes from this transversal and supra-national sector.
\end{abstract}

Keywords: Argentina, Sensitive technologies, Dual use, Space Technology

\section{ARGENTINA NO ESPAÇO: POLÍTICA INTERNACIONAL EM RELAÇÃO A POLÍTICA TECNOLÓGICA E O DESENVOLVIMENTO INDUSTRIAL}

\section{Resumo}

O artigo de reflexão presente tem como principal propósito desenvolver e compreender de forma analítica o caminho de meio século de tecnologia espacial que vem se desenvolvendo na República Argentina. O estilo da avaliação podese tipificado como um caso de desenvolvimento de tecnologia capital intensiva, considerada "sensível" pelos países centrais. A tecnologia capital intensiva, implica níveis importantes da industrialização, mas também de poder militar. O 
objetivo geral consiste em expor qual é a maneira em que uma serie de rotura políticas e econômicas transformaram os alinhamentos que deveriam orientar a trajetória do desenvolvimento da tecnologia espacial, assim como a sua aplicação no uso civil e no bélico. Igualmente o artigo explica como funciona a disciplina das Relações Internacionais a partir de uma política tecnológica, se permitindo mostrar os principais resultados deste setor transversal e supranacional.

Palavras Chave: Argentina, Tecnologias sensível, Uso Dual, Tecnologia Espacial

\section{Introducción}

El presente trabajo se propone analizar medio siglo de desarrollo de tecnología espacial en la Argentina como un caso de desarrollo de una tecnología capital intensiva1, considerada "sensible" por los países centrales, en contexto de país semiperiférico. El objetivo es mostrar de qué manera una serie de rupturas políticas y económicas transformaron los objetivos que debían orientar la trayectoria del desarrollo de tecnología espacial, conspirando contra los procesos de aprendizaje y acumulación de capacidades y competencias, con el saldo de atrasos y dilapidación de recursos materiales y humanos ${ }^{2}$. Conjuntamente, se busca interpretar el rol de la política internacional a partir de la política tecnológica analizada.

La debilidad de las políticas tecnológicas — falta de competencias para la formulación de políticas capaces de definir metas y objetivos de manera realista - la falta de un entorno institucional capaz de crecer, diversificarse, aumentar su complejidad y producir enraizamientos con el sector público de Investigación y Desarrollo (I+D) y el sector empresarial con capacidades potenciales, junto con las iniciativas formales e informales de coerción de los países centrales, han sido la regla en la historia del desarrollo tecnológico, y en especial el caso espacial argentino (Hurtado de Mendoza, 2010), y en consecuencia,

Entendemos al concepto capital-intensivo como grandes inversiones de capital durante un período de tiempo largo, y para obtener resultados a largo plazo. Dentro de esta definición, no sólo entra la inversión líquida, sino la mano de obra especializada que requiere de varios años de estudio de grado, posgrado, y luego entrenamiento específico para el desarrollo de habilidades que le permitan conocer, producir, y reproducir la tecnología, así como la transmisión de dichos conocimiento y la formación de nuevos cuadros técnicos. Ver Importación de tecnologías capital-intensivas en contextos periféricos: el caso de Atucha I (1964-1974)" (Fernández, 2010).

2 Argentina contó con niveles importantes de desarrollo de tecnología espacial, incrementalmente desde la década de 1960. Militares y civiles estuvieron involucrados en distintos desarrollos y proyectos. 
este se ha visto eclipsado desde su desarrollo incremental a partir de la década de 1960, hasta la destrucción del Cóndor II, que ha virado a partir de la creación de la Comisión Nacional de Actividades Espaciales (CONAE) y ha comenzado una nueva etapa institucional de acceso al espacio en la República Argentina (Blinder, 2015a). Entendemos la política espacial como toda política tendiente a lograr los objetivos espaciales, ya sea adquirir satélites o cohetes lanzadores.

Este artículo abordará la cuestión de la semiperiferia para entender la importancia del lugar en el cual se desarrolla la tecnología, e indagará acerca del concepto hegemonía en la política internacional. Prosigue el artículo describiendo el concepto tecnología y buscando su comprensión en el contexto de política doméstica y exterior en el cual se desenvuelve. Se desarrolla luego una breve descripción del contexto industrial argentino, y de la tecnología espacial. Seguidamente, el presente trabajo realiza un análisis con respecto a la Argentina y la cuestión de la tecnología espacial, estudiando los períodos 1960-1982, 1982-1991, 1991-2003, y 2003 hasta el presente. Se describen los condicionamientos estructurales locales e internacionales y los logros de la tecnología espacial, en su vinculación con la coyuntura económico-política, local y global. Se reflexiona e indaga acerca de la dimensión contextual en la cual el desarrollo espacial se da como política tecnológica e industrial y, finalmente, se explicitan algunas reflexiones y propuestas a partir de las disquisiciones abordadas.

\section{La cuestión de la semiperiferia}

Existen algunos componentes que configuran un sistema internacional, entre los que sobresalen la hegemonía en el sistema mundial y la reconfiguración del Capital en función del Estado (Ghotme, 2006). Un sistema mundial es un "modo económico [que] se basa en el hecho de que los factores económicos operan en el seno de una arena mayor de lo que cualquier entidad política puede controlar totalmente" (Wallerstein, 2005: 491), yendo más allá de las estructuras de los Estados Nación, y forma espacios centrales, semiperiféricos y periféricos. Según esta clasificación, decimos que son Estados centrales aquellos más poderosos -económica y políticamente en el sistema internacional. Esto quiere decir que son los países más dinámicos y desarrollados, económica y tecnológicamente, pero que también presentan una estructura estatal con capacidad de influir en el sistema de Estados, con un importante poder militar y diplomático. Son periféricas aquellas unidades estatales que no tienen poder económico ni político y por ello son poco relevantes económicamente y en el sistema interestatal. No obstante ello, son parte del mismo y aportan principalmente materias primas y productos primarios. Son semiperiféricos aquellos países de 
la periferia con capacidad industrial, científica y tecnológica. Los Estados semiperiféricos tienen más relevancia política en el sistema interestatal que los periféricos.

Una característica muy importante de la semiperiferia, desde el punto de vista de los países centrales, es que al tener capacidad industrial y desarrollo científico y tecnológico, demandan tecnología de los países centrales que en última instancia podría resultar competencia para su mercado. Tecnologías capital intensivas como la nucleary la espacial son impulsadas sólo por países centrales y minoritariamente por la semiperiferia. Son tecnologías sensibles que conforman mercados codiciados oligopólicamente por los países centrales. Por lo tanto, el desarrollo de este tipo de tecnologías para el sistema mundial podría resultar desestabilizador. Un país de la semiperiferia podría ser en el futuro aquel que se convierta en el centro del sistema internacional. En este proceso de ascenso en la jerarquía de los Estados, la tecnología, además del capital, es un factor central (Wallerstein, 2005). Adicionalmente, existe una paradoja en el sistema internacional en la que hay un aumento del gasto militar generalizado, sumado a la intensificación de los programas nucleares en todo el mundo. Todo esto produce desequilibrios en la cuestión de la seguridad global ${ }^{3}$ (Cubillos et al., 2013).

Consideramos aquí que aquel Estado o conjunto de unidades estatales que sean el centro del sistema mundial son los hegemónicos. El orden político, militar e institucional derivado de esto será el orden hegemónico:
"Las instituciones proveen maneras de enfrentar los conflictos y minimizar, de ese modo, el uso de la fuerza. Hay un reforzamiento potencial en las relaciones materiales de poder subyacentes a cualquier estructura, en el cual el más fuerte puede dar una paliza al débil si piensa que es necesario. Pero la fuerza no debe ser utilizada con el fin de asegurar el dominio del fuerte hasta el límite de que el débil acepte las relaciones de poder prevalecientes como legítimas. Esto lo puede hacer el débil si el fuerte ve su misión como hegemónica $y$ no meramente dominante o dictatorial, esto es, si desea hacer concesiones que puedan asegurar la aquiescencia del débil respecto a su liderazgo y si puede expresar su liderazgo en términos de intereses universales o generales, más que

Si se tiene en cuenta los países que poseen capacidades nucleares militares, y sistemas de misiles para alcanzar los objetivos, se puede apreciar por qué las potencias que tienen dichos sistemas no quieren compartirlo: para no tener competencia y para mantener su poder militar (Cubillos et al., 2013). 
como mero apoyo a sus propios intereses particulares" (Cox, 1994: 145).

Mientras tanto, "la noción de hegemonía como una combinación de poder, ideas e instituciones hizo posible tratar algunos de los problemas en la teoría de dominación estatal como la condición necesaria de un orden internacional estable" (Cox, 1994: 154). En otro texto, Robert Cox se pregunta si el concepto hegemonía es un término unívoco en el ámbito de la política internacional, y señala que mayormente se lo utiliza para hablar del poder de unos países sobre otros, de dominación o supremacía (Cox, 1996: 60). Sin embargo, el autor explica que hegemonía internacional es el predominio de un Estado entre otros, pero es expresado a través de normas universales de comportamiento para los Estados, instituciones en donde estas últimas reposan, y un modo económico, social y cultural de producción. Esa hegemonía se ejerce desde el centro hacia la periferia. ¿Qué es exactamente el centro, la semiperiferia, y la periferia del sistema internacional?

En línea con las definiciones de Cox y Wallerstein, creemos que países centrales del sistema internacional son aquellos que, como unidades estatales, ejercen la hegemonía política, institucional, económica, social, y cultural. Está respaldada por un poder político-militar propio o de alianza de alcance mundial. Esto quiere decir que en el mundo post-soviético dicha potencia unipolar fue EE. UU., por lo menos durante la década de 1990; y, en la década de 2000 y principios de la de 2010, la preferencia es de un mundo que tiende a presentar otros polos de poder: además de EE. UU., China, Rusia, India, Japón, países europeos especialmente Alemania, Francia y Gran Bretaña. Algunos señalan también a Brasil, o Sudáfrica, aunque no hay consenso entre los diferentes analistas acerca de los criterios para determinar cuál Estado entra y cuál no en un polo de poder y por qué. Con todo, decimos aquí que países del centro son EE. UU. y sus principales aliados del orden internacional de posguerra hasta la caída de la Unión Soviética, y con polos emergentes que no llegan aún a ser países centrales, como China, por ejemplo, con gran potencial económico, político y cultural.

Son países periféricos aquellos que no ejercen la hegemonía y pueden aceptar o no el orden hegemónico. Son países semiperiféricos aquellos que, siendo esencialmente no centrales - no tienen poder suficiente para formar y forjar reglas - presentan algunas características diferentes a los periféricos, es decir, tienen alguna relevancia estratégica para el sistema, ya sea económica, cultural, política, o tecnológica. A los efectos de las definiciones conceptuales necesarias, la Argentina, Brasil, China o la India, Egipto, Irán, Israel, o Sudáfrica son ejemplos de países semiperiféricos, 
que no siendo casos absolutos $y$ equiparables, sí tienen grados de influencia, variable y creciente, en el sistema internacional.

\section{Argentina, un país espacial}

¿Qué sucedió en la Argentina en materia de tecnología espacial, teniendo en cuenta que es un país semiperiférico, por lo que no es forjador de reglas, pero presenta cierta relevancia política y económica en el sistema internacional? Desde la década de 1960 la Argentina tuvo un desarrollo incremental de capacidades satelitales y lanzadores (Hurtado, 2010), hasta el Proyecto Cóndor. Dicho proyecto era un vector balístico, por lo que constituía una tecnología de uso dual, siendo en principio un proyecto de la Fuerza Aérea Argentina que derrotada en la Guerra de Malvinas de 1982 contra Gran Bretaña, y habiendo perdido gran parte de su material bélico y pilotos — buscó desarrollar capacidades disuasivas contra la potencia colonial ocupante del territorio en disputa (Blinder, 2011). Llegado el gobierno democrático de Raúl Alfonsín en 1983, se procuró continuar con el desarrollo del Cóndor II en su carácter dual, mas quedó durante este gobierno virtualmente asfixiado por las dificultades económicas que atravesaba el país. Por esta razón, se recurrió al financiamiento externo, cuyo resultado redundó en la participación de empresas europeas, y de Estados del Medio Oriente como Egipto o Irak, lo cual repercutió diplomáticamente durante el gobierno de Carlos Menem, que a partir de las presiones internacionales que recibía, del ahogo presupuestario por una alicaída economía y un viraje de su política exterior, decidió cancelar el proyecto (Blinder, 2015a). A partir de la cancelación del Cóndor II se creó en consecuencia y directa la CONAE, cuyo objetivo era enmarcar la política espacial en la política exterior.

Pero existe aquí una variable que atraviesa transversalmente todo este proceso político de desarrollo tecnológico, que es la económica. La economía o el modelo económico implementado ha determinado en general los lineamientos políticos locales, los proyectos institucionales, y los desarrollos tecnológicos. Hasta el golpe militar de 1976, la Argentina implementaba políticas tendientes a desarrollar capacidades endógenas, pero a partir de la dictadura la economía tendió —con diferentes tiempos y gradaciones - hacia la extranjerización neoliberal. Por ello, se abandonó la idea del desarrollo de tecnologías de punta nacional. Estos procesos acompañaron a la variable política y la diplomática.

En la presidencia Perón entre 1946 y 1955 primaban conceptos ideológicos vinculados al nacionalismo militar, con una marcada orientación de desarrollo industrial propio y nacionalismo tecnológico (Raccanello, 2013). Distintos desarrollos vinculados a lo 
aeroespacial como el avión caza a reacción - desarrollo a la altura de los producidos entre las principales potencias - como fue el Pulqui, desarrollo de cohetes como el Tábano o el Martín Fierro. Todos estos productos se daban en un contexto en el cual primaba en la formación de los cuadros políticos y militares el concepto de Nación en Armas y de Movilización Total, lo que estratégicamente se operacionalizaba como producción industrial para la Defensa, pero también como industrialización. Durante la presidencia de Frondizi, que se extendió hasta ser derrocado por un golpe militar entre 1958 y 1962, se creó la CNIE (Comisión Nacional de Investigaciones Espaciales) con Teófilo Tabanera como presidente. Luego, a pesar de los sucesivos golpes militares y llamados a elecciones interrumpidos, se continuó el desarrollo, sobre todo cohetes sonda para la investigación atmosférica, con combustible sólido. Los Cohetes Sonda Alfa Centauro, Beta Centauro, Gamma Centauro, Orión, Canopus, son ejemplos concretos de estos desarrollos. Con el cohete Canopus II se envió al "mono Juan" fuera de la atmósfera y se lo retornó con vida, lo que significaba un logro científico y sobre todo una capacidad técnica de vanguardia. También se envió al espacio al "Ratón Belisario" y se lo retornó con vida a la Tierra. Igualmente se desarrollaron los Cohete Rigel, Castor, y Tauro (De León, 2008). Los satélites en Argentina son un asunto que empieza a consolidarse hacia la década de 1990, y tienen que ver con otro proceso de institucionalización de la política espacial, y un contexto internacional que demandaba los servicios que estos dispositivos proveían. Satélites argentinos como Lusat 1, Víctor-1, SAC-A, SAC-B para la observación astronómica, SAC-C para la observación terrestre, SAC-D Aquarius para la observación salinidad marina y también utilizable con otros instrumentos para la observación terrestre, nanosatélites, SAOCOM para las comunicaciones son la muestra del progreso en este campo, como geoestacionarios producidos localmente Arsat-1 y Arsat 2 por la empresa de comunicaciones satelitales estatal, Arsat.

La participación de la industria en el PBI nacional argentino ha tenido un rol preponderante y en evolución hasta llegar a fines de la década de 1970, cuando empieza a decaer. Como muestran los dos siguientes gráficos, tomando en cuenta el volumen total, el país ha ganado capacidades en su industria hasta comenzar un proceso de desindustrialización, pero en términos relativos, el rol de este sector comienza a recuperar vigor a partir de la década del 2000, más tomando en cuenta el PBI per cápita. Si observamos el crecimiento de la actividad espacial hacia los años sesenta, veremos cómo este acompaña al crecimiento de actividad industrial. Los años ochenta y noventa, con una caída de la actividad industrial, también presentaron un decrecimiento de las actividades espaciales, excepto el Cóndor, 
Inversión Estatal en Misiones Satelitales

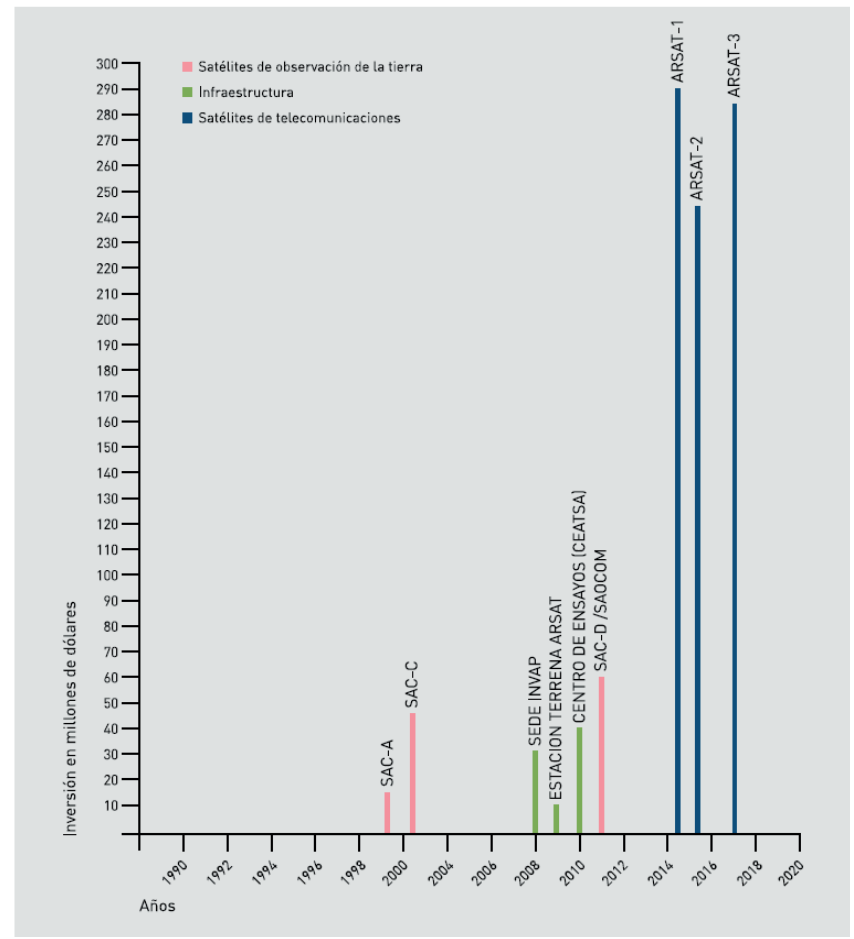

Fuente: El sector espacial argentino. Instituciones, empresas y desafíos (2014).

que se encontró posteriormente 1960-1982

desfinanciado primero por problemas económicos y luego también de orden político internacional. Comenzado el nuevo siglo, se puede ver un crecimiento de la actividad espacial que acompañó un aumento de la actividad industrial ${ }^{4}$.

Durante las dos décadas iniciales de desarrollo de tecnología espacial en Argentina, se comienzan a integrar a un conjunto de sectores estratégicos para impulsar la construcción de capacidades y entornos institucionales,

$4 \quad$ La actividad industrial nunca consiguió lograr los niveles que supo tener la Argentina desde la década de 1950. Se puede percibir, comparando los gráficos año a año, la relación existente entre intervención del Estado para conseguir objetivos tecnológicos, y actividad de la industria. Toda actividad tecnológica convive con una actividad industrial, puesto que ella necesita saberes, ingeniería, adquisión y desarrollo de materiales, producción en serie, etc. 


\section{GRÁFICO 3}

PARTICIPACIÓN DEL PIB INDUSTRIAL EN EL TOTAL

(En porcentaje)

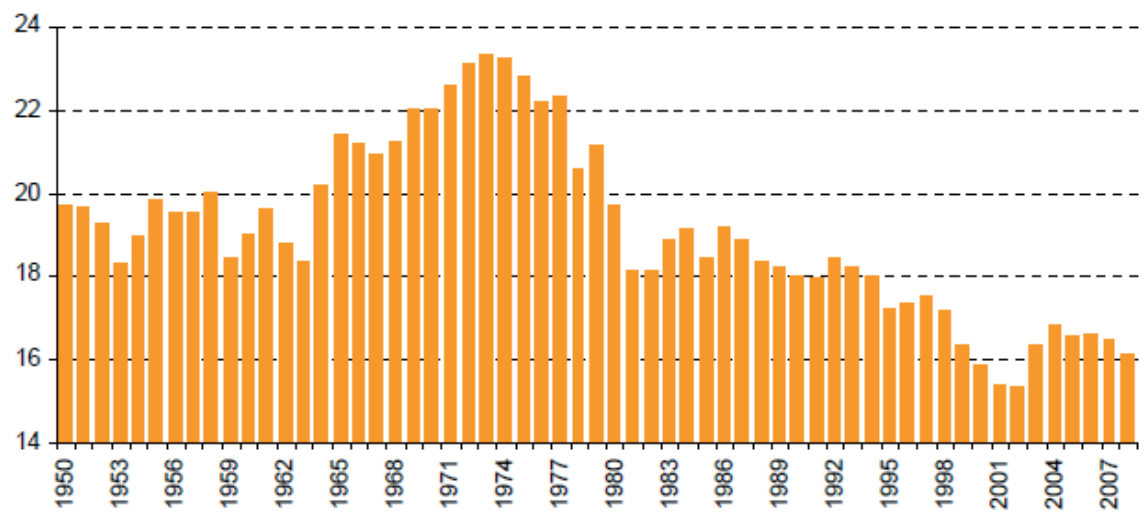

\section{GRÁFICO 4}

EVOLUCIÓN DEL PIB INDUSTRIAL PER CÁPITA

(En pesos a precios constantes de 1993)

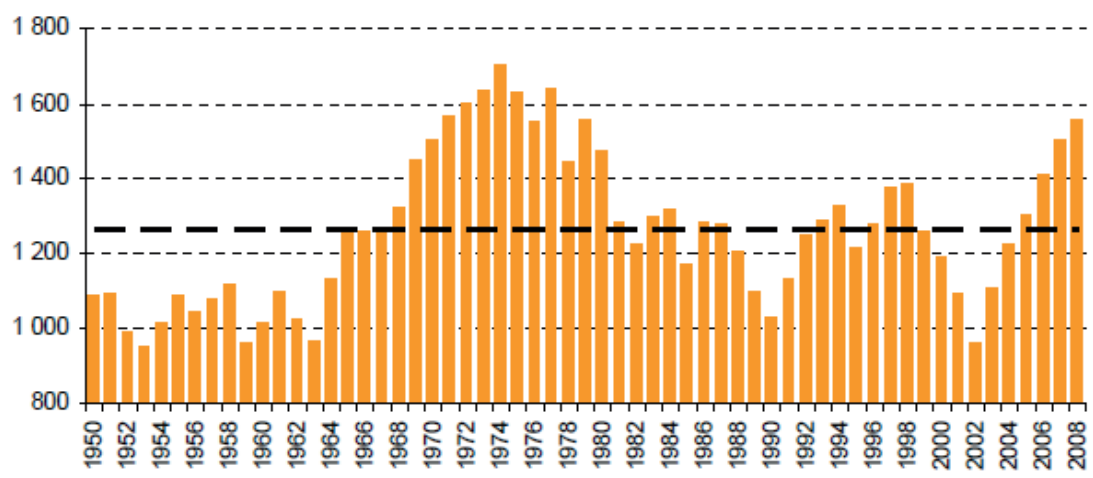

Fuente: Kosacoff, B. (2010). CEPAL. Marchas y contramarchas de la industria argentina (1958-2008).

capaces de desplegar procesos del proceso de industrialización; de desarrollo de un conjunto de (ii) favorecer la participación de tecnologías capital intensivas que firmas nacionales, objetivo que son consideradas estratégicas para también significaba el mejoramiento los países centrales. En el caso de la de sus capacidades tecnológicas; Argentina, los objetivos de esta apuesta (iii) responder a las demandas de son: (i) potenciar el segundo estadio modernización del sector militar; y 
(iv) construir un lugar de prestigio y liderazgo regional (Hurtado, 2010).

En relación con el desarrollo de tecnología espacial, la unidad de este período viene dada por: (i) el desarrollo de cohetes de complejidad creciente para investigación científica $y$, desde comienzos de los setenta, de un lanzador satelital con expectativas de proyección regional; (ii) la dependencia del desarrollo de tecnología espacial del sector militar (CNIE $^{5}$, Fuerza Aérea); y (iii) la estrategia de desarrollo incremental de tecnología para cohetes, que promueven procesos acumulativos de aprendizaje y knowhow y la participación creciente en estos procesos de empresas locales estatales, mixtas y privadas, en contexto de políticas económicas nacionales orientadas a la segunda fase del proceso de industrialización; y (iv) se promueven componentes ideológicos vinculados a la búsqueda de la autonomía tecnológica y del liderazgo regional.

\section{2-1991}

La evolución del escenario político y económico, caracterizado en la Argentina por un alto grado de inestabilidad, transformó a comienzos de los años ochenta las motivaciones y los objetivos, así como el sentido político y económico del desarrollo de tecnología espacial. La política económica de la última dictadura puso fin a cuatro décadas de industrialización para dar inicio a un patrón de acumulación que tuvo como núcleo dinamizador la valorización financiera y la apertura y desregulación de la economía. Mientras el país se desindustrializa, un sector minoritario de la cúpula militar en el gobierno promueve valores industrialistas, entre ellos la necesidad de la autonomía tecnológica en sectores estratégicos, como el nuclear, el energético o el espacial. En este contexto, la guerra de las Malvinas actúa como catalizador que justifica el abandono del proyecto de desarrollo incremental de capacidades nacionales por un proyecto de misil balístico con tecnología importada, nueva estrategia que aceleraría los procesos de acceso a tecnologías de punta.

La unidad de este período viene dada por: (i) el objetivo de desarrollar un misil balístico de alcance medio con un componente estratégico — amenazar la posición británica en las islas Malvinas -, y componentes comerciales y de política exterior relacionados con compromisos comerciales a cambio de la participación en el financiamiento del proyecto de países de medio

Comisión Nacional de Investigaciones Espaciales, era la institución encargada de los asuntos espaciales en la Argentina, y fue manejada por la Fuerza Aérea Argentina. 
oriente; (ii) la dependencia del proceso de toma de decisiones del sector militar (CNIE, Fuerza Aérea); (iii) se abandonan dos décadas de desarrollo incremental para impulsar el proyecto de desarrollo de un misil balístico con la participación dominante de empresas extranjeras, que aportan la tecnología, en contexto de desindustrialización y de componentes ideológicos dominantes vinculados a posiciones nacionalistas confrontativas motivadas por la derrota militar en la guerra de las Malvinas; y (iv) se suman expresiones difusas y descontextualizadas sobre metas relacionadas a la autonomía tecnológica (Blinder, 2015a).

\section{1-2003}

La nueva transformación que tendrá lugar a comienzos de los años noventa estará motivada por la profundización del patrón de valorización financiera impulsada por una política económica de desregulación, apertura de la tecnología y "achicamiento del Estado". La necesidad de obtener préstamos de los organismos internacionales de financiamiento llevó a implementar una política exterior de alineamiento incondicional a la potencia hegemónica, estrategia que resignificó los proyectos de desarrollo de tecnologías "sensibles", como las desarrolladas por los sectores nuclear y espacial, pero también sectores estratégicos como hidrocarburos o agrario.

La unidad de este período viene dada por: (i) el abandono del proyecto de desarrollo de un misil balístico para pasar al desarrollo de tecnología satelital con asistencia norteamericana (NASA), proceso guiado por la actualización periódica de un plan espacial de mediano plazo que se propone el desarrollo de capacidades autónomas, la prestación de servicios con aplicaciones económicas y ambientales, con proyección comercial hacia la región; (ii) el paso del desarrollo de tecnología espacial de la esfera militar a la civil con la creación de la CONAE (Ministerio de Relaciones Exteriores); (iii) el abandono de los vínculos con las empresas extranjeras que aportaban el know-how para dar paso a la participación de empresas y capacidades locales, con un lugar protagónico de la empresa INVAP', en un contexto de desindustrialización selectiva y privatización de empresas públicas; (iv) se promueven componentes ideológicos difusos vinculados a grupos de INVAP con antecedentes en el sector nuclear que sostienen una retórica de acumulación de capacidades, que se enfrentan a componentes ideológicos dominantes

\footnotetext{
6 INVAP (Investigación Aplicada) es una empresa argentina que se dedica al desarrollo de tecnologías de punta, industrial, nuclear, espacial.
} 
en la diplomacia argentina vinculados a la perspectiva conocida como "realismo periférico", posición explícitamente funcional a la política económica.

\section{3-Presente}

El proceso político que se inicia en 2003 propone el abandono gradual de la matriz neoliberal y la recuperación de una política económica de industrialización con presencia estratégica del Estado, que se propone, a largo plazo, la transformación de la matriz productiva a través de la incorporación de valor agregado. Adaptadas al nuevo contexto, emergen componentes de política tecnológica afines al primer ciclo de industrialización y se vuelve a considerar la tecnología espacial como componente estratégico para impulsar la construcción de capacidades y entornos institucionales capaces de desplegar procesos de desarrollo de un conjunto de tecnologías capital intensivas con capacidad de dinamizar otros sectores de la economía. Una diferencia crucial con la década de los setenta es que, si bien las tecnologías espaciales siguen siendo de interés para los países centrales, pueden considerarse tecnologías maduras (Ruttan, 2006).

Las tecnologías sensibles parecen establecer una línea divisoria también entre capacidades políticas y éticas. Por lo tanto, esta ideología conservadora y jerárquica de cómo debe funcionar el mundo, propone un equilibrio estático que relega a los países no centrales que quieren tener la posibilidad de entrar en mercados de alto valor agregado —aún con estrategias diplomáticas multilaterales, dialoguistas, pacifistas, desarrollistas y antimilitaristas - a la marginalidad tecnológica y, por lo tanto, a la dependencia de las potencias, que se mueven en un sistema y un orden internacional que ellas mismas crearon. En algún sentido, podría concluirse que, los países en desarrollo como la Argentina deberían ser capaces de articular su política tecnológica y su política exterior de tal forma que, logrando consolidar en la arena internacional una caracterización de país confiable - lo que también significa no ser considerado una amenaza en el terreno de la competencia (Blinder, 2015b) comercial en sectores de alto valor agregado -, lograran ganar la suficiente autonomía como para avanzar en el desarrollo autónomo de tecnologías sensibles que finalmente le permitirían acceder a algún nicho de mercado.

Si bien esta afirmación parece contradictoria, no lo es en virtud de las escalas temporales en juego. Es decir, si la Argentina lograra un lanzador satelital ${ }^{7}$ en los próximos años, un balance histórico permitiría asumir que

Cohete lanzador. 
este logro le habría llevado alrededor de cinco décadas desde que los primeros países centrales lograron desarrollar los primeros inyectores, tiempo suficiente para que esta tecnología pasara a ser considerada madura, es decir, que entrara en la fase de aplanamiento de la curva de aprendizaje y, por lo tanto, en el estadío en que deja de ser de interés prioritario para las potencias. Retrospectivamente, la carrera por un lanzador satelital significaría para la Argentina una carrera por lograr formar parte del grupo de los primeros países de la semiperiferia en acceder tempranamente-relativoalostiempos de la periferia - a una tecnología en transición de transformarse en madura, que por lo tanto comienza a dejar de ser prioritaria para las potencias, pero que en las economías semiperiféricas pueden ser un factor de dinamismo económico a la vez que un logro estratégico en términos de liderazgo o prestigio regional.

Si a la Argentina le resulta más rentable, en términos de competencia económica, pagar por el envío de un satélite propio en cohete ajeno, que hacer las costosas inversiones en investigación y desarrollo local —que llevan años y a veces sin éxito -, entonces es más barato no desarrollar la propia tecnología. La afirmación "no es necesario para el país desarrollar una tecnología tan compleja y cara como la espacial" es una afirmación aislada de cualquier posibilidad de disponer de una política tecnológica. La necesidad es una de las muchas razones por las cuales se puede buscar el desarrollo de una tecnología. Se puede desarrollar una tecnología por razones de prestigio internacional, para ganar liderazgo, para contar con capacidad de disuasión, por cuestiones de mercado y comerciales, o para crear una industria o serie de industrias en un país o región e impulsar procesos de desarrollo económico.

Reaparece el objetivo de favorecer la participación de firmas nacionales y el objetivo de liderazgo regional presente en los setenta es reemplazado por formulaciones políticas incipientes que conciben estrategias de colaboración con Brasil $^{8}$ y buscan resignificar la tecnología espacial como uno de los vehículos aptos para la integración política y económica regional. La unidad de este período viene dada por: (i) la continuidad del desarrollo de tecnología satelital heredada de los noventa, así como de su entorno institucional, guiados por la producción de satélites de complejidad

En la historia argentina reciente, a partir del gobierno Néstor Kirchner, un giro en la política económica que buscó desarrollar el mercado interno y una política de integración regional cuyo objeto era la consolidación de un mercado regional, reduciendo las asimetrías con respecto a las potencias (Rapoport, 2012). 
tecnológica incremental —observación y telecomunicaciones $-y$, como novedad crucial, la incorporación al (la recuperación para el) plan espacial del proyecto de desarrollo incremental de un lanzador satelital a partir de capacidades locales en un contexto de reindustrialización; (ii) la continuidad de la dependencia civil, aunque la CONAE pasa de depender, sobre el final de este período, del Ministerio de Relaciones Exteriores al Ministerio de Planificación; (iii) se profundiza y diversifica la participación de empresas y capacidades locales con el objetivo de conformar un sector de empresas nacionales proveedoras en un contexto de acelerado crecimiento económico (por lo menos hasta 2009) y se comienza a pensar en la exportación de tecnología satelital a países de la región; (iv) se promueven componentes ideológicos vinculados a la búsqueda de la autonomía tecnológica —satélites de observación y de telecomunicaciones y lanzadores nacionales - y, de forma ambigua, a la integración regional que a veces toma la forma de búsqueda del liderazgo regional.

El siguiente gráfico muestra el incremento presupuestario en la Argentina con respecto al desarrollo satelital nacional. Si bien toma en cuenta a partir del año 2003 correspondiente a la presidencia Kirchner -, el recorte permite divisar la clara vocación del Estado de inversión pública en esta tecnología, llegándose a números considerables. El cuadro que prosigue al gráfico, muestra la articulación entre el sector público y privado, y las empresas que

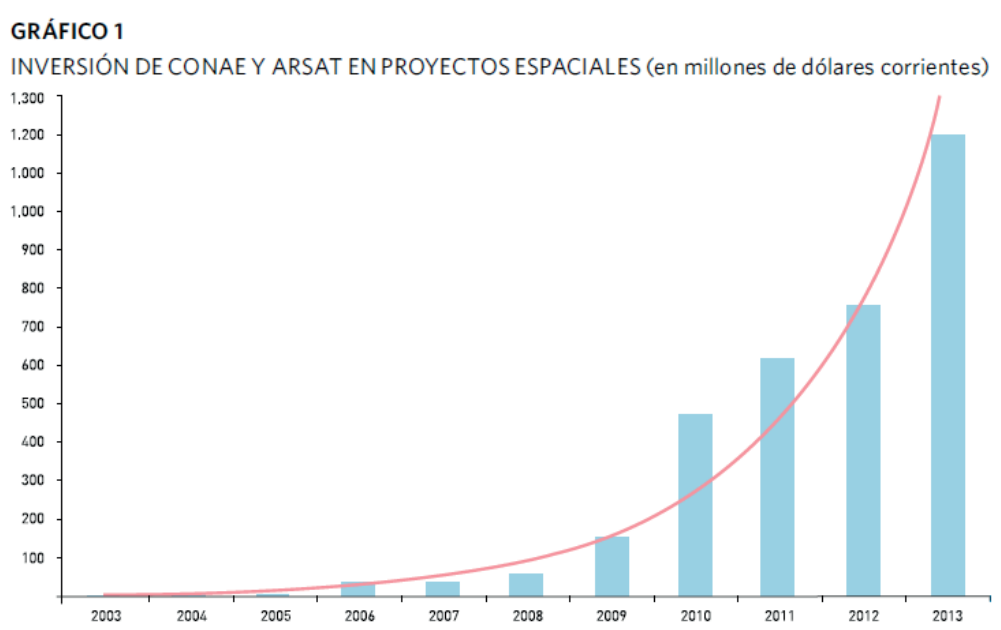

Fuente: Elaboración propia en base a datos de CONAE y ARSAT.

Fuente: El sector espacial argentino. Instituciones, empresas y desafíos (2014). 
han nacido al calor del desarrollo del sector a partir del año 2003. Empresas privadas han desarrollado proyectos en conjunto con el Estado.

La economía argentina ha experimentado desde la década de 1990 una fuerte desindustrialización, y un crecimiento de los sectores financieros y agrarios, como muestra el siguiente cuadro. Aquí lo que se puede observar es que si bien la estructura industrial no ha tenido un gran salto, ha recuperado junto a otros sectores su dinamismo, y uno de ellos ha sido el sector de la industria de tecnología espacial. En el primer cuadro se puede observar la estructura del sector industrial y cómo sufrió ese proceso de decaimiento, hasta reiniciar su recuperación, por políticas activas por parte del Estado, el cual se ve el rol de la ingeniería en general. En el segundo cuadro, se observa la evolución global de la economía y sus efectos en los años de políticas económicas neoliberales, y la época posterior con orientación hacia la producción.

\section{Articulación política}

Para los países semiperiféricos como la Argentina, su éxito económico se juega en su capacidad de articular su política tecnológica y su política

\begin{tabular}{|c|c|c|c|c|c|c|c|c|c|}
\hline EMPRESA & UBICACIÓN & CEO & $\begin{array}{l}\text { AÑODE } \\
\text { FUNDACÓN }\end{array}$ & $\begin{array}{l}\text { ACTIVIDAD } \\
\text { PRINCIPAL }\end{array}$ & $\begin{array}{l}\text { PRINCIPALES } \\
\text { CLIENTES }\end{array}$ & $\begin{array}{l}\text { CANTIDAD } \\
\text { DE } \\
\text { EMPLEADOS }\end{array}$ & $\begin{array}{l}\text { CANTIDAD DE } \\
\text { INGENIEROSY } \\
\text { EMPLEADOS } \\
\text { TECNICOS }\end{array}$ & $\begin{array}{l}\text { PROYECTOS } \\
\text { ESPECIALES } \\
\text { ENLOSQUE } \\
\text { PARTICIPO }\end{array}$ & $\begin{array}{l}\text { DESARROLIO } \\
\text { DE PRODUCTOS } \\
\text { PROPIOS }\end{array}$ \\
\hline ARSULTRA & $\begin{array}{l}\text { Capital } \\
\text { Federal }\end{array}$ & $\begin{array}{l}\text { Juan } \\
\text { Martín } \\
\text { Semegon }\end{array}$ & 2012 & $\begin{array}{l}\text { Desarrollo de } \\
\text { tecnología } \\
\text { aeroespacial e } \\
\text { industrial } \\
\text { para aplicaciones }\end{array}$ & $\begin{array}{l}\text { CONAE; } \\
\text { VENG; } \\
\text { Tenaris- }\end{array}$ & 7 & 6 & $\begin{array}{l}\text { SAC-D } \\
\text { /Aquarius }\end{array}$ & $\begin{array}{l}\text { Diseño de } \\
\text { enclosures }\end{array}$ \\
\hline ASCENTIO & Córdoba & $\begin{array}{l}\text { Esteban } \\
\text { Carranza }\end{array}$ & 2008 & $\begin{array}{l}\text { Ingeniería, Desarrollo } \\
\text { de Software, } \\
\text { Operación } \\
\text { de Sistemas de Alta } \\
\text { Disponibilidad }\end{array}$ & CONAE & 77 & 76 & $\begin{array}{l}\text { SAC-D/ } \\
\text { Aquarius; } \\
\text { SAOCOM; } \\
\text { SABIAMAR; } \\
\text { TRONADOR }\end{array}$ & No \\
\hline DTA & Córdoba & $\begin{array}{l}\text { Javier } \\
\text { Siman }\end{array}$ & 2006 & $\begin{array}{c}\text { Desarrollo de } \\
\text { equipamientos de alta } \\
\text { tecnología para la } \\
\text { industria aeroespacial } \\
\text { y militar }\end{array}$ & $\begin{array}{l}\text { CONAE, } \\
\text { INVAP, } \\
\text { FADEA, } \\
\text { FFAA }\end{array}$ & 31 & 20 & $\begin{array}{l}\text { SAC-D } \\
\text { /Aquarius; } \\
\text { SAOCOM; } \\
\text { IA 63 } \\
\text { PAMPA }\end{array}$ & $\begin{array}{c}\text { Controlador del } \\
\text { radar de apertura } \\
\text { sintética; splitters y amplificadores de rf. }\end{array}$ \\
\hline $\begin{array}{l}\text { MECÁNICA } \\
14\end{array}$ & Bariloche & $\underset{\text { Martín }}{\text { Breerton }}$ & 1987 & $\begin{array}{l}\text { Mecanizados de alta } \\
\text { precisión. }\end{array}$ & $\begin{array}{l}\text { INVAP; } \\
\text { YPF; } \\
\text { Tenaris; } \\
\text { CONAE; } \\
\text { etc }\end{array}$ & 19 & 2 & $\begin{array}{l}\text { SAC-A, SAC-B, } \\
\text { SAC-C, SAC-D, } \\
\text { SAOCOM; } \\
\text { ARSATIy } \\
\text { ARSAT ॥ }\end{array}$ & $\begin{array}{l}\text { Válvulas esféricas } \\
\text { puentes producción } \\
\text { puentes inyección. } \\
\text { Accesorios especiales para } \\
\text { ind. petrolera }\end{array}$ \\
\hline SADE & $\begin{array}{l}\text { Capital } \\
\text { Federal }\end{array}$ & $\begin{array}{c}\text { Altor } \\
\text { García }\end{array}$ & 2011 & $\begin{array}{l}\text { Desarrollo integral de } \\
\text { proyectos de ingeniería } \\
\text { de alta tecnolggia con } \\
\text { orientación al sector } \\
\text { nuclear y espacial. }\end{array}$ & $\begin{array}{l}\text { CONAE, } \\
\text { CNEA Y } \\
\text { NASA }\end{array}$ & 31 & 15 & $\begin{array}{l}\text { Programa } \\
\text { Acceso al } \\
\text { Espacio de } \\
\text { CONAE }\end{array}$ & $\begin{array}{l}\text { Ruedas "mecanum", las cuales permiten } \\
\text { realizar plataformas de movimiento } \\
\text { omnidireccional y bancos de ensayos } \\
\text { para calificación de componentes } \\
\text { mecánicos de uso espacial. }\end{array}$ \\
\hline STI & Córdoba & $\begin{array}{l}\text { Oscar } \\
\text { Alberto } \\
\text { Ignazi }\end{array}$ & 1991 & $\begin{array}{l}\text { Servicios de } \\
\text { Ingeniería }\end{array}$ & $\begin{array}{l}\text { CONAE } \\
\text { Y VENG }\end{array}$ & 110 & 71 & $\begin{array}{l}\text { SAOCOM, } \\
\text { SARAT, } \\
\text { SARE, } \\
\text { TRONADOR }\end{array}$ & $\begin{array}{l}\text { Dos desarrollos para el Proyecto SARE: } \\
\text { uno de ellos vinculado a laconfiguración } \\
\text { computacional distribuida y, } \\
\text { el otro, a las comunicaciones } \text { liter } \\
\text { satelitales y satélites - tierra. }\end{array}$ \\
\hline SUR & $\begin{array}{l}\text { Capital } \\
\text { Federal }\end{array}$ & $\begin{array}{l}\text { Alberto } \\
\text { Pérez } \\
\text { Cassinelli }\end{array}$ & 2006 & $\begin{array}{l}\text { Desarrolloy } \\
\text { aplicaciones de } \\
\text { tecnologías } \\
\text { geoespaciales. }\end{array}$ & $\begin{array}{l}\text { CONAE, STI, } \\
\text { ScanTerra, } \\
\text { Secretaria de } \\
\text { Ambiente }\end{array}$ & 25 & 20 & $\begin{array}{l}\text { SAOCOM, } \\
\text { SARAT, } \\
\text { SARE, } \\
\text { TRONADOR }\end{array}$ & $\begin{array}{l}\text { Productos de Software } \\
\text { Geoespacial Suri. }\end{array}$ \\
\hline
\end{tabular}

Fuente: El sector espacial argentino. Instituciones, empresas y desafíos (2014). 


\begin{tabular}{|c|c|c|c|}
\hline \multicolumn{4}{|c|}{$\begin{array}{c}\text { Cuadro } 7 \\
\text { Valor agregado } \\
\text { (Tasas de crecimlento promedio anual) }\end{array}$} \\
\hline Sectores & $1990-1998$ & $1998-2002$ & $2002-2010$ \\
\hline Intensivos en ingenleria sin autor & 6,40 & $-13,68$ & 11.62 \\
\hline Auto & 10,09 & $-16,20$ & 12,70 \\
\hline Total intensivos en Ingenierla & 8,06 & $-14,88$ & 12,13 \\
\hline Allmentos, bebidas y tabaco & 4.97 & $-8,38$ & 8,35 \\
\hline Otros Intensivos en recursos naturales & 4.67 & $-8,78$ & 5,36 \\
\hline Total intensivos en recursos naturales & 4,84 & -8.55 & 7.12 \\
\hline Total Intensivos en trabajo & 2,89 & 2,21 & 8.25 \\
\hline TOTAL INDUSTRIA & 5,00 & -7.55 & 8,12 \\
\hline
\end{tabular}

\begin{tabular}{lrrr}
\multicolumn{5}{c}{ PIB: Tasas de crecimiento promedio anual } & \\
\hline Agricuitura & $1990-1998$ & $1998-2002$ & $2002-2010$ \\
Minerta & 3,48 & $-0,65$ & 4,85 \\
Industria & 6,79 & 0,48 & 1,04 \\
Electricidad & 5,00 & $-7,55$ & 8,12 \\
Construccion & 7,68 & 1,49 & 5,54 \\
Comercio & 10,24 & $-16,67$ & 14,55 \\
Transporte & 6,24 & $-9,07$ & 9,52 \\
Establecimientos nnanciercs & 8,33 & $-3,57$ & 12,22 \\
Servicios comunales, sociales y personales & 7,48 & $-3,90$ & 5,79 \\
Pis total & 2,81 & $-0,48$ & 5,11 \\
\hline
\end{tabular}

Fuente: CEPAL (2013). La industria argentina frente a los nuevos desafíos y oportunidades del siglo XXI.

exterior de tal forma que, logrando consolidar en la arena internacional una caracterización de país confiable - lo que también significa no ser considerado una amenaza en el terreno de la competencia comercial en sectores de alto valor agregado -, conseguirán ganar la suficiente autonomía como para avanzar en el desarrollo autónomo de tecnologías sensibles que finalmente le permitirían acceder a algún nicho de mercado de alto valor agregado, además de "derramar" capacidades tecnológicas hacia otros sectores. Si bien esta afirmación parece contradictoria ser confiable a la vez que competidor -, no lo es en virtud de: (i) las escalas temporales en juego; y (ii) de posibles estrategias de negociación.

Con respecto a (i), que la Argentina logró acumular capacidades para el desarrollo de satélites con relativa autonomía hace alrededor de una década, yqueseencuentraactualmente en proceso de investigación y desarrollo de un lanzador ${ }^{9}$ para dominar todo el ciclo tecnológico ${ }^{10}$, se puede inferir a partir de las experiencias de los países

\footnotetext{
$9 \quad$ El Tronador 2 es un vehículo lanzador de satélites desarrollado por la Argentina, con grados importantes de avance.

10 Poder hacer satélites y poder lanzarlos con cohetes de tecnología propia.
} 
centrales, que países semiperiféricos con desarrollo de tecnologías de propósito general ${ }^{11}$, a pesar de llegar décadas más tarde, cuando la tecnología hubiera comenzado el proceso de transformación en tecnología madura, estas tecnologías resultan conflictivas por la competencia económica y también por cuestiones de seguridad internacional. Adicionalmente, el avance de estas tecnologías le pavimenta el camino para poder desarrollar otras vinculadas a este proceso industrial, lo que permite ampliar la cadena de valor. Más aún, se puede pensar que a partir de un adelanto así, se produce un efecto derrame y multiplicador hacia otras áreas entrelazadas con el primer desarrollo, como la apertura de carreras universitarias para el desarrollo de tecnologías, materiales, y ciencias vinculadas al sistema, empresas de base tecnológica, laboratorios, un sector comercial vinculado al mercado externo e interno, y un sector público que gestione la política tecnológica ${ }^{12}$ en sus facetas económica e industrial pero también en su regulación y negociación diplomática internacional. De esta manera, entendiendo que toda política de defensa es también una política exterior, y que toda política tecnológica implica la regulación de las tecnologías en instituciones internacionales, la gestión de la diplomacia es clave para llevarlas a cabo con éxito.

Con respecto a (ii), negociar tecnología satelital con la NASA a cambio de abandonar el proyecto de misil balístico resignándose a perder las ingentes inversiones que se habían concretado, mientras que, en paralelo, se consolida una política exterior que intenta construir confiabilidad, puede haber sido tranquilizador para los países centrales. La posibilidad de que en la arena diplomática se acepte - siempre de forma ambigua e inestable - que la Argentina vuelva sobre un proyecto de lanzador satelital demuestra que la articulación de política tecnológica y política exterior puede abrir una brecha en la lógica económica de la tecnología de punta que solo busca proteger mercados oligopólicos.

\section{Conclusiones}

Varias cuestiones podrían ponerse sobre la mesa a la hora de tratar de

11 Tecnología de propósito general es aquella de aplicabilidad general, y que a partir de ella se producen otros desarrollos, como por ejemplo la tecnología del motor a combustión, nuclear, o espacial (Ruttan, 2006).

12 Existen diversos estudios que analizan la relación entre la vinculación de estos actores con el Estado, y algunos estudian específicamente la relación con tecnologías de uso dual. Es el caso de la industria de aviación en Suecia (Eliasson, 2010) o el caso de las industrias tecnológicas de avanzada en EE. UU., entre las cuales se encuentran las tecnologías duales como la nuclear, espacial o internet, y que tuvieron sus inicios en la promoción y participación directa del Estado, no de los capitales privados (Mazzucato, 2013). 
sintetizar y reflexionar acerca de lo vertido en estas páginas. La propuesta de analizar medio siglo de desarrollo espacial argentino, considerado tecnológicamente como "sensible", o una actividad cuyo resultado es potencialmente proliferante, $y$ consecuentemente peligrosa para los países centrales en términos de seguridad internacional, pero también por cuestiones económicas y de desarrollo.

En esa línea, los cambios en la vida política e institucional del país y los cambios de orientación en la política económica —que definió distintos objetivos de desarrollo industrial o tecnológico - modificaron no solamente los resultados concretos, sino también los procesos de aprendizaje y acumulación de capacidades de los actores involucrados en la tecnología.

En efecto, podemos decir que la cuestión tecnológica trabajada en el presente artículo nos recuerda su faz dual: y al ser desarrollada esta tecnología capital intensiva en un país semiperiférico como la Argentina implica competidores de países que cuentan con el dominio de dicha tecnología, por lo que pierden el monopolio de su producción militar.

Los cohetes y satélites son peligrosos en cuanto su potencial uso por parte de Fuerzas Armadas, y el control de cuáles son los países pasibles de comprar esa tecnología se pierde cuando alguno de ellos puede fabricarla.

La ciencia, la tecnología y la política local e internacional, son conceptos absolutamente entrelazados. Cuando pensamos en tecnología, estamos pensando en un sistema tecnológico, que excede ampliamente a los aparatos o productos técnicos, cuya cristalización redunda en poder nacional.

Consecuentemente, toda política tecnológica es también una política de desarrollo y una política exterior, porque implica también la cristalización de ese poder nacional a partir de la política tecnológica. La tecnología es de uso dual, y la guerra emplea tecnología. La guerra es la continuación de la política por otros medios. Por lo tanto, no puede pensarse una política tecnológica sin su contexto político local o internacional.

Principalmente, está la cuestión de las presiones de las potencias que sufren los países de la periferia, al desarrollar este tipo de tecnologías, y especialmente la trabajada aquí. En efecto, la Argentina sufrió de planteamientos por parte de EE. UU. para abandonar el desarrollo espacial, y conducirse hacia una política pacífica, que estuviera contenida en el marco de los regímenes internacionales de no proliferación de armas de destrucción masiva. Por consiguiente, el motivo por el cual la 
República Argentina transformó su política fue por los vínculos con esos países y sus inquietudes de política exterior $^{13}$.

Otro factor importante para reflexionar es la cuestión de la debilidad institucional e inercia institucional durante las transformaciones. La Argentina careció de instituciones científico-tecnológicas robustas, que le permitieran una continuidad en el tiempo de ciertos desarrollos, favoreciendo a burocracias civiles y militares que trabajaron sobre la coyuntura.

Un cambio de política exterior, a partir de los sucesivos cambios de política interna por alternancia civil-militar, y gobiernos sensibles a las presiones de distintos grupos de interés -locales y globales- constituyó además la trayectoria de la política espacial, hasta la creación de la CONAE que fue logrando institucionalidad.

Conjuntamente, es menester destacar los procesos de aprendizajes, incluida la experiencia adquirida en el área nuclear, así como la continuidad de algunos actores, como la empresa de alta tecnología INVAP o la Comisión Nacional de Energía Atómica, y otros. Estos actores fueron acumulando experiencia y ayudaron a sostener y reproducir los conocimientos tecnológicos argentinos, aun en circunstancias difíciles.

Finalmente, la Argentina comienza a dominar la tecnología satelital cuatro décadas más tarde que la puesta en órbita del primer satélite y todavía no domina la tecnología de lanzadores satelitales. ¿Qué significa madurez tecnológica y cómo se aplica este concepto al caso estudiado?

1. El proyecto Cóndor significó un quiebre que abandonó una estrategia de acumulación incremental de capacidades locales en materia de vectores, que se implementó a lo largo de casi dos décadas (desde aproximadamente 1960 hasta fines de los años setenta), para pasar a una organización compleja, con actores estatales y privados, nacionales y extranjeros, y financiamiento local y extranjero, que apostó a la transferencia de tecnología de punta de países centrales. Sin mencionar la cuestión de Malvinas y la decisión de contar con material bélico, y sin tomar en cuenta la cuestión presupuestaria de un país ahogado financieramente tras las políticas económicas adoptadas por la

13 En los años de la década del 1990 la política exterior de la Argentina estaba basada en lo que se denominó Realismo Periférico, cuyo objetivo principal era no confrontar con las potencias. El Cóndor II resultaba un problema para las relaciones entre EE. UU. y los argentinos, y por lo tanto una confrontación no necesaria, en las cuales se perdía mucho más por confrontar que por no hacerlo (Escudé, 1992). 
dictadura derrotada en una guerra, podemos destacar una coyuntura mundial de desarrollo económico y tecnológico en mutación, cuya característica principal radica en la trasnacionalización productiva, la liberalización financiera, y un cambio en la forma de producir (que comienza en la década de 1970). Varios técnicos a cargo del Cóndor Il tenían una formación profesional en EE. UU. y, además, formaban parte de la más "tecnológica" de las fuerzas, cuya conducción requería el conocimiento del estado del arte de las cuestiones de su arma, lo que pudo haber sido el disparador para salir del pensamiento de la autarquía en materia de desarrollo de capacidades propias. De cualquier modo, el Cóndor significaba un salto tecnológico significativo que no pudo concretarse por la falta de planificación política y financiera.

2. Para el desarrollo del Cóndor se creó una arquitectura empresarial compleja, que combinó la creación de empresas nacionales (creadas ad hoc) para interaccionar con empresas extranjeras. La idea de esta organización empresarial era hacer posible mecanismos de transferencia tecnológica, saberes y locación para la producción. De acuerdo con los testimonios, se habrían logrado ciertos niveles de transferencia y se habrían producido procesos de aprendizaje y formación de recursos humanos, aun sin haber concluido el misil.

3. El Estado tuvo un papel central en el proceso de desindustrialización argentino, pero en distintos niveles. El Estado en la dictadura comenzó un proceso para liberalizar la economía, pero en algunos aspectos tuvo una política industrialista, como con respecto al Cóndor II o al sector nuclear. EI papel del empresariado nacional fue pobre. El proceso más relevante fue la consolidación de prácticas de transferencia de recursos públicos a manos de grupos empresariales concentrados, lo que algunos autores llamaron la "patria contratista". En este sentido, el proyecto Cóndor no fue una pieza central de esta dinámica, como sí lo fue el desarrollo nuclear, aunque la empresa Techint ${ }^{14}$, una de las más beneficiadas de este escenario económicoqueseconfigura durante la última dictadura, participó como contratista principal en la construcción de las instalaciones de Falda del Carmen ${ }^{15}$.

4. Para ingresar insumos del misil, el gobierno de facto se valió de

14 Techint es una de las empresas multinacionales más grandes de la Argentina, y gran contratista del Estado.

15 Es el lugar donde se montó el centro de desarrollo secreto del misíl Cóndor II por parte de la Fuerza Aérea. Hoy en día las instalaciones son utilizadas por la Comisión Nacional de Actividades Espaciales. 
una estructura ilegal alrededor del aeropuerto de Ezeiza, creando estructuras empresarias paralelas que operaban en conjunto con la Fuerza Aérea. Durante el gobierno de Alfonsín, el Estado no controló el proyecto, por lo que siguió bajo el control de la Fuerza Aérea. La crisis económica y financiera heredada de la dictadura no favoreció mucho la participación del empresariado argentino. $\mathrm{Y}$ durante el gobierno de Menem el papel del Estado fue abiertamente desindustrializador.

5. El Estado argentino desmanteló el proyectoCóndor,yabocóaldesarrollo de tecnología espacial enmarcada en una agencia espacial, que va a tener un proceso de desarrollo de lanzador satelital Tronador 2, así como varios desarrollos satelitales como la serie SAC, SAOCOM, O ARSAT. Todos estos desarrollos se ven potenciados a mediados de la década del 2000 , con distintos niveles de éxito, acompañando el crecimiento del país y el repunte industrial.

A partir de todo lo estudiado, quedan abiertas varias líneas de investigación, entre las cuales destacamos las siguientes:

- La relación entre política exterior y otras tecnologías sensibles en contextos periféricos, para aportar nuevos datos a la presente investigación.

- La relación entre política exterior y el desarrollo de tecnologías no sensibles, para aportar nuevos datos acerca del comportamiento entre estas dos variables, en la cual el desarrollo tecnológico no afecta temas de la agenda de seguridad.

- La relación histórica entre política exterior y tecnología en los países centrales, para aportar luz a la comprensión de qué política utilizaron estos países, antes de volverse potencias militares, económicas, y tecnológicas.

- Políticas públicas en ciencia y tecnología, especialmente estudios comparativos de políticas espaciales de otros países de la semiperiferia, las posibilidades de cooperación internacional, y sus relaciones con los países centrales.

En cualquier caso, el recurrente $-y$ obvio - argumento de los actores que protagonizaron la trama elaborada en el presente trabajo tendió a justificar sus acciones y decisiones políticas y a minimizar sus errores y consecuencias a futuro.

A modo de esquematización, los militares sugieren que sus acciones fueron en pos de la defensa y la soberanía nacional, y consideran que el gobierno de Menem liquidó el patrimonio tecnológico nacional. Los radicales, en sus términos, consideran que sostener el proyecto tenía que ver con la soberanía en términos de defensa y desarrollo tecnológico. Por el contrario, el gobierno que gestionó 
durante el decenio de 1990 considera exitosas sus posturas, pero alocadas, extravagantes, y extemporáneas las de sus predecesores, cuyas acciones, según ellos, no hacían más que erosionar de forma creciente la confiabilidad argentina, y sus posibilidades reales de inserción en el mundo. ¿Por qué extemporáneas y extravagantes? Porque en su imaginario conceptual los actores políticos de la década menemista ven en gobiernos anteriores posturas que oscilan entre la irracionalidad y la mala praxis de un país tercermundista en un contexto de Guerra Fría y, por supuesto también, actitudes inconducentes para el nuevo orden imperante tras la caída del bloque soviético.

En este tenor, recalcan una y otra vez el concepto de país confiable y los gestos internacionales para concretarlo, como los tratados, las alianzas extraOTAN, o la cancelación de tecnologías conflictivas. Bajo esta visión, poseer vectores es solamente para grandes potencias. Eso significa no sólo que tienen el poder, la capacidad económica y el estatus internacional para poseerlas, sino que se supone que tienen además las capacidades políticas y técnicas que legitiman su posesión y empleo.

Eso significa, elementalmente, que los países con débiles procesos de desarrolloy, por lo tanto, sin la capacidad económica ni el poder político necesarios no estarían preparados para la producción y el empleo responsable de las tecnologías sensibles. Si este tipo de tecnologías pueden considerarse imprescindibles en la selección de un tipo de sendero hacia el desarrollo económico, una inferencia es que la "no confiabilidad" en el campo de la proliferación y la seguridad también aparece como un obstáculo insalvable hacia el crecimiento económico. Pero también de un poder industrial aplicable al desarrollo del poder militar ofensivo y disuasivo: esa es la principal característica de una tecnología sensible.

\section{Referencias}

Drewes, L. (2014). El sector espacial argentino: Instituciones referentes, proveedores y desafíos. Benavidez: ARSAT.

Blinder, D. (2015a). Argentina space: ready for launch, Space and Defense Journal, 8 (1). Journal of the United States Air Force Academy. Eisenhower Center for Space and Defense Studies. 34-46.

Blinder, D. (2015b). Hacia una política espacial en la Argentina. Revista Iberoamericana de Ciencia Tecnología y Sociedad (CTS), 10 (29). 65-89.

Blinder, D. (2011). Tecnología misilística y sus usos duales: aproximaciones políticas entre la ciencia y las Relaciones Internacionales en el caso del V2 alemán y el Cóndor II argentino. Revista Iberoamericana de Ciencia Tecnología y Sociedad (CTS), 6 (18). 9-33. 
CEPAL. (2013). La industria argentina frente a los nuevos desafíos y oportunidades del siglo XXI. Santiago de Chile: CEPAL.

Corigliano, F. (2003). La Dimensión Bilateral de las Relaciones entre Argentina y Estados Unidos durante la Década de 1990: El ingreso al paradigma de las 'Relaciones Especiales'. En: Escudé, C. (Ed.). Historia General de las Relaciones Exteriores de la República Argentina, Parte IV, Tomo XV. Buenos Aires: GEL.

Cox, R. (1994). Fuerzas sociales, estados y órdenes mundiales: más allá de la teoría de las relaciones internacionales. En: Vásquez, J. Relaciones Internacionales. El pensamiento de los clásicos. Barcelona: Limusa.

Cox, R. (1996). Gramsci, Hegemony, and International Relations: An Essay in Method, En: Cox and Sinclair (eds.), Approaches to World Order. Cambridge: Cambridge University Press.

Cubillos, A., Garay, C., Carrier, A., \& Hernández, D. (2013). Desarrollo nuclear: ¿Otoño o primavera para la proliferación en actores estatales? Revista de Relaciones Internacionales, Estrategia y Seguridad, 8 (2), 143-165.

De León, P. (2008). Historia de la Actividad Espacial en la Argentina. Edición del Autor: Buenos Aires.
Eliasson, G. (2010). Advanced public procurement as industrial policy. The aircraft industry as a technical university. New York: Springer.

Escudé, C. (1992). Realismo Periférico: Fundamentos para la nueva política exterior Argentina. Buenos Aires: Planeta.

Fernández, J. (2010). Importación de tecnologías capital-intensivas en contextos periféricos: el caso de Atucha I (1964-1974). Revista Iberoamericana de Ciencia Tecnología y Sociedad (CTS), 6 (16). 9-37.

Ghotme, R. (2006). Orden, Des-Orden y Antiorden Mundial. Problemas de Relaciones Internacionales. Revista de Relaciones Internacionales, Estrategia y Seguridad, 1 (2). 75-703.

Hurtado de Mendoza, D. (2010). La ciencia argentina. Un proyecto inconcluso. 1930-2000. Buenos Aires: Edhasa.

Kosacoff, B. (2010). Marchas y contramarchas de la industria argentina (1958-2008). Santiago de Chile: CEPAL.

Mazzucato, M (2013). The Entrepreneurial State. Debunking public vs. private sector myths. London: Anthem Press.

Raccanello, M. (2013). Industrias Aeronáuticas y Mecánicas del Estado y la lógica de la política económica peronista. América Latina en la historia económica, 20 (2), 177-221. 
Rapoport, M. (2012). Historia económica, política y social de la Argentina, 1880-2003. Buenos Aires: Emece.

Ruttan, V. (2006). Is War Necessary for Economic Growth? Military
Procurement and Technology Development. Oxford: Oxford University Press.

Wallerstein, I (2005). El moderno sistema mundial, Tomo I. Méjico: Siglo XXI. 